\title{
Johann Christoph Gottsched und die Olmützer "Monathlichen Auszüge alt, und neuer Gelehrten Sachen" (1747-1748)
}

\section{Johann Christoph Gottsched and the Olomouc's „Monthly Excerpts from Old and New Erudition" (1747-1748)}

Sabine Voda Eschgfäller

\begin{abstract}
The article deals with the influence of Johann Christoph Gottsched on the so-called first Austrian learned society of Enlightenment, the "Societas eruditorum incognitorum in terris Austriacis" and its scientific journal named "Monathliche Auszüge alt, und neuer Gelehrten Sachen". Gottsched was not just a member of the learned society in Olomouc, but also took part in the activities oft he journal: in 1748 for instance he published an essay on the development of Austrian literature since the Middle Ages and supervised the production of the publication in Leipzig, since the journal had to move away from Moravia because of political issues. But even before he had been an important factor for the choice of (German) language used in the journal.
\end{abstract}

\section{Keywords}

Johann Christoph Gottsched; Societas eruditorum incognitorum in terris Austriacis; Monathliche Auszüge alt, und neuer Gelehrten Sachen; Enlightenment; Moravia; learned society; Habsburgic Monarchy 
Eine intensivere Auseinandersetzung mit der Geschichte der „Societas incognitorum eruditorum in terris Austriacis "1 begann die österreichische Geschichtswissenschaft im Laufe des 19. Jahrhunderts, wobei sich v.a. der Brünner Landeshistoriker Christian Ritter d'Elvert als Initiator dieses Prozesses hervorgetan hat. D'Elvert beschäftigt sich in seiner Geschichte des Deutschtums in Österreich-Ungarn ${ }^{2}$ und im „Notizen-Blatt“ ${ }^{\text {“3 }} \mathrm{u}$. a. mit der oft als erste aufklärerische Gelehrte Gesellschaft in den österreichischen Ländern bezeichneten Sozietät und dem Adelsgeschlecht, dem ihr Gründer, der Freiherr Joseph von Petrasch entstammte; in den folgenden Jahrzehnten wird der mährische Kommunalpolitiker sowohl in Publikationen deutschsprachiger als auch tschechischer (bzw. tschechoslowakischer) Wissenschaftskreise wiederholt zitiert. ${ }^{4}$ Im Vordergrund der meisten Artikel zum Thema Societas incognitorum stehen die Eckdaten der Gründung dieser Vereinigung, v. a. aber eine Auflistung ihrer Beisitzer und Mitglieder, die den Sonderstatus dieser Sozietät in der mährischen Provinz unterstreicht, sowie ihr bereits genannter Gründer, der slawonische ehemalige Adjutant von Prinz Eugen. ${ }^{5}$ Immer wird - fast beiläufig - auch erwähnt, dass die Societas incognitorum ein eigenes Rezensionsjournal ${ }^{6}$ herausgegeben habe und festgehalten, wie lange (bzw. eigentlich eher wie kurz) die Erscheinungsdauer der Publikation gewesen war. Hingewiesen wird meist auch darauf, welchen Fachgebieten man sich in dem Gelehrten Journal mit dem etwas holprig anmutenden Titel „Monathliche Auszüge alt, und neuer gelehrten Sachen"7 gewidmet hatte. Genauer besprochen werden aber die fast durchwegs anonym darin erschienenen Titel in der Regel nicht. Es wurde bislang auch nur selten (und wenn ja, nur punktuell) versucht, die Rezensionen bzw. Auseinandersetzungen mit damals aktuellen natur- und geisteswissenschaftlich Themen mit dem europäischen wissenschaftlichen Diskurs der Mitte des 18. Jahrhunderts zu vernetzen. ${ }^{8}$ Dieser Bei-

1 Im weiteren Verlauf des Textes wird der Namen der Gelehrtengesellschaft mit „Societas incognitorum“ abgekürzt.

2 S. D'Elvert, Christian (1884): Zur Geschichte des Deutschthums in Oesterreich-Ungarn, mit besonderer Rücksicht auf die slavisch-ungarischen Länder. Brünn: Rohrer Verlag, S. 495.

3 D'Elvert, Christian (1866): Zur mährischen Adelsgeschichte. XI. Die Freiherrn von Petrasch. S. 33-36. In: Notizen-Blatt der historisch-statistischen Section der kaiserlich-königlich mährisch-schlesischen Gesellschaft zur Beförderung des Ackerbaus, der Natur- und Landeskunde. Beilage der Mittheilungen. Brünn: Verlag des Vereines für die Geschichte Mährens und Schlesiens, S. $33 \mathrm{f}$.

4 Vgl. auch D'Elvert, Christian (1884): Zur Geschichte des Deutschthums in Oesterreich-Ungarn, mit besonderer Rücksicht auf die slavisch-ungarischen Länder. Brünn: C. Winiker, S. 495.

5 Vgl. Schram, Wilhelm (1894): Josef Freiherr von Petrasch und die „Gelehrte Gesellschaft der Unbekannten in Olmütz“. In: Notizenblatt des Vereines für die Geschichte Mährens und Schlesiens, Brünn: Verlag des Vereines für die Geschichte Mährens und Schlesiens, Nr. 10, S. 89-91.

6 Zur Geschichte und Funktion von Rezenzionsjournalen s. Habel, Thomas (2007): Gelehrte Journale und Zeitungen der Aufklärung. Zur Entstehung, Entwicklung und Erschließung deutschsprachiger Rezensionszeitschriften des 18. Jahrhunderts. Bremen: Edition lumière (Presse und Geschichte. Neue Beiträge. Hrsg. von Holger Böning, Michael Nagel und Johannes Weber; Band 17)

7 Statt des vollständigen Titels der Publikation wird im Folgenden die Abkürzung „MA“ verwendet.

8 Dieser Aufsatz ist im Rahmen eines von der Philosophischen Fakultät der Palacký-Universität in Olomouc unterstützten Projektes entstanden, das sich dezidiert mit dem Gelehrten Journal beschäftigt, welches die Societas incognitorum herausgegeben hat („Monathliche Auszüge alt, und neuer gelehrten Sachen. První časopis rakouského osvícenství v evropském kontextu“; FPVČ-Projektnummer 452101391) 
trag zur Geschichte der MA versucht, zumindest einen Aspekt des Gelehrten Journals aufzuzeigen, in welchem ein bedeutender Gelehrter der deutschen Aufklärung agiert. Johann Christoph Gottscheds Beteiligung an der Societas incognitorum ${ }^{9}$ und seine Artikel in den MA sollen illustrieren, wie die Olmützer Gelehrtengesellschaft sich in ihren Entscheidungen orientierte, will heißen, für welche (aufklärerischen) Orientierungspunkte sie sich entschied und damit einhergehend, inwieweit die sich bereits im Fluss befindende Aufklärung in den deutschsprachigen Ländern außerhalb der habsburgischen Landesgrenzen in die sich in Mähren-Schlesien bildenden aufklärerischen Initiativen hineinspielte.

\section{Gottsched und die Gründung der Societas incognitorum}

Joseph von Petrasch hatte sich nach seinen Ausbildungs- und Militärjahren 1743 erneut in Olmütz niedergelassen und dort ein Palais bezogen, das er von seiner Mutter geerbt hatte. In diesem, direkt am Oberring gelegenen Gebäude hatte er schon einige Zeit wöchentliche Treffen für Interessierte abgehalten, die über Wissenschaft und Kunst diskutieren oder selbst referieren wollten. Diese Zusammenkünfte, wenige Meter vom Olmützer Rathaus entfernt, verursachten beinahe augenblicklich das Misstrauen nicht nur der geistlichen (von Jesuiten dominierten) Obrigkeit, sondern auch der Stadtobersten, die die Vereinigung einer Untersuchung unterziehen ließen, um freimaurerische Aktivitäten auszuschließen. ${ }^{10}$

Petrasch verfügte aus seiner militärischen Vergangenheit und aus der Zeit seiner Bildungsreisen, die ihn durch ganz Europa geführt und in Kontakt mit gelehrten Gesellschaften und einflussreichen Gelehrten gebracht hatte, über Möglichkeiten, sich beispielsweise von Seiten des Wiener Hofs oder durch namhafte Persönlichkeiten Unterstützung zu besorgen. Vermutlich rechnete er bereits bei der Gründung der Societas incognitorum aufgrund seiner Kenntnis der geistigen Atmosphäre in der Stadt an der March mit Schwierigkeiten. Dementsprechend lud er zum Gründungsakt einige ausgewählte Beisitzer ein, die von höfischer Seite anerkannt waren und/oder in der Welt der Wissenschaft und Kunst bereits über einen unbestritten guten Leumund verfügten. Betrachtet man die Liste der Personen, die an der Wiege der Gelehrtengesellschaft standen, finden sich nicht nur Offiziere bzw. Beamte, sondern auch gelehrte Geistliche,

9 Mit dem Thema der Mitgliedschaft Gottscheds in der Olmützer Gelehrtengesellschaft beschäftigte sich Rieck bereits 1973, ohne jedoch näher auf dessen Motivation und Artikel einzugehen. S. Rieck, Werner (1973): Gottsched und die Societas incognitorum in Olmütz. In: Deutsche Akademie der Wissenschaften Berlin, Ost (Hrsg.): Forschungen und Fortschritt, Nr. 40, Berlin: Akademie Verlag, S. 83-85.

10 Petrasch verfügte über eine umfangreiche Bibliothek, die, ebenso wie die Sammlung des Domprobstes Giannini, als Grundlage für Forschungen im Umkreis der Societas incognitorum und der MA dienen konnte. Petrasch finanzierte die MA (und die damit verbundenen Kosten) durch sein Privatvermögen. Vgl. hierzu auch Wögerbauer, Michael/ Pokorný, Jiří (2015): Barocke Buchkultur in den böhmischen Ländern. In: Gastgeber, Christian/ Klecker, Elisabeth (Hrsg.): Barock. Graz: Akademische Druck-und Verlagsanstalt, S. 383-426. 
sowohl aus protestantischen wie auch aus katholischen Ländern und über die Landesgrenzen bzw. Sprachgrenzen hinweg. ${ }^{11}$

Gottsched hatte Petrasch bis dahin nicht persönlich kennengelernt; es ist auch nicht bestätigt, ob es tatsächlich je zu einer Begegnung zwischen den beiden Männern gekommen ist. Nichtsdestotrotz wendet sich der Ideen- und Geldgeber der MA am 29. November 1746, sozusagen am Vorabend der Gründung der Gelehrtengesellschaft, an den Leipziger Professor für Logik und Metaphysik und lädt ihn durchaus selbstbewusst ein, seinem Kreis beizutreten. Das Ziel, Gottsched zu gewinnen, verbindet sich bei Petrasch wohl auch mit der Absicht, einen erfahrenen „Zeitungsmann“, Stilisten und Autoren mit ins Boot zu holen. Den Verfasser des „sterbenden Cato“ versucht er auf ähnliche Weise zu beeindrucken, wie die Verfasser von Beiträgen über die Societas incognitorum seither, um ihr Thema zu rechtfertigen: durch Name-dropping und zwar, wie anzunehmen ist, in einer wohl überlegten Art und Weise, wobei die Reihenfolge der Namen anscheinend illustrieren sollte, wie „international“ die Gelehrtengesellschaft aufgestellt war. Als erste Persönlichkeit, die bereits gewonnen wurde, wird der florentinische Geschichtsprofessor Antonio Francesco Gori ${ }^{12}$ angeführt, weiter der ebendort lebende Professor für Kirchengeschichte Giovanni Lami, dann - für den österreichischen Raum - Jean de Baillou, der ab 1748 das Wiener Naturalienkabinett leitete und der lothringische Offizier in habsburgischen Diensten François de la Motte. Darauf wird noch der Domprobst der Olmützer Mauritzkirche, Franz Gregor Giannini, erwähnt, der im Laufe der Zeit zu einem der wichtigsten Mitarbeiter und vielleicht auch Opponenten von Petrasch avancieren sollte. ${ }^{13}$ Auf ihn folgen dessen Bruder, Ernst Friedrich Giannini, der ungarische Historiker und (lutherische) Theologe Matthias Bel, der St. Pöltner Priester und Bibliothekar Raimund Duelli und schließlich ein bekannter und vor allem anerkannter Name der deutschsprachigen Geschichtswissenschaft, der Göttinger Professor Johann David Köhler. Der vorletzte Name, der in diese Passage des Schreibens eingetragen wird, ist der des Priesters und Dichters Nicolaus Christian Sander, ehe Petrasch die als überzeugendes Argument aufgebaute Reihe mit einem Petrus Ciner(i)us schließt, ein Pseudonym, das er bis dahin für seine eigenen Arbeiten benutzt hatte. ${ }^{14}$

Im Brief an Gottsched erläutert Petrasch noch vor der eigentlichen Umsetzung des Publikationsprojektes die grundlegende Absicht, die durch die Arbeit an der Zeitschrift resp. durch die Verbreitung der MA verwirklicht werden sollte; der spätere Besitzer des

11 Vgl. Kollmann, Vitězlav (1998): Societas incognitorum a olomoučtí zednáři. In: Zprávy Vlastivědného muzea v Olomouci, Nr. 276, S. 1-8.

12 Gori hatte sich beispielsweise durch die Entzifferung von etruskischen Inschriften hervorgetan, sowie als Kenner römischer Skulpturen. Er war seit 1737 ebenso Mitglied der namhaften „Accademia della Crusca“, die als älteste Sprachgesellschaft überhaupt gilt. Damit ist er ein weiteres der Mitglieder der Olmützer Sozietät, das ebenfalls nicht nur renommiert, sondern innerhalb des Wissenschaftsbetriebes gut vernetzt und renommiert war. S. Sandys, John Edwin (2010): A History of Classical Scholarship. Charleston: Nabu Press, S. 384.

13 Vgl. Králík, Oldřich (1947): Olomoucká Societas incognitorum. Olomouc: Lidové závody tiskařské a nakladatelské, S. 23.

14 Vgl. Wondrák, Eduard (1972): Die olmützer „Societas incognitorum“. Zum 225. Jubiläum ihrer Gründung und zum 200. Todestag ihres Gründers. In: Ischreyt, Heinz u.a. (Hrsg.): Die Aufklärung in Ost- und Südosteuropa. Aufsätze, Vorträge, Dokumentationen. Köln/ Wien: Böhlau Verlag, S. 215-228. 
Anwesens Neuschloß ${ }^{15}$ bei Butschowitz in Mähren erklärt - und wirbt für sein Vorhaben folgendermaßen:

„Ich weis gar wohl, daß dergleichen Bande: welche die Gleichheit. Deren Gemüthern, oder Amts, oder Vorsatz zu einem Unternehmen begehren, sich bey mir, von Euer Hochedelgebornen, nicht hoffen lässet; denn obgleich, ich mit Denenselben, in dem Fall, einerley Beschäftigung, übernommen, daß ich alle Mühe, Sorge, ja Haab und Guth, daran zu wenden, mich entschlossen habe, um die Wissenschaften, um freyen Künste, in unserm Deutschland; oder vielmehr, in denen Oesterreichischen Landen, gleichwie Sie, in Sachßen, schon längst gethan haben, empor zu bringen, finde dennoch eine so große Ungleichheit unserer Fähigkeit, daß ich nicht weis ob Dieselben eines Neulings Freundschaft annehmen, und ihn wiederum der Ihrigen werden theilhaftig machen wollen." ${ }^{16}$

Die patriotische Aufgabe, der sich Petrasch stellen will, indem er die Ideen der Aufklärung auch von Mähren aus in den österreichischen Ländern verbreitet, schöpft also aus dem Vorbild Gottscheds, der dies in Sachsen nach Petraschs Dafürhalten bereits aktiv tat. Die Verehrung, die er dem arrivierten Gelehrten und Schriftsteller in seinem ersten Schreiben an ihn erweist, geht einher mit der Versicherung (die noch an einer anderen Stelle des Briefes wiederholt wird), dass die MA auf einer soliden finanziellen Grundlage, nämlich dem Privatvermögen Petraschs, stehen; damit will er wohl die in der damaligen Zeit oft angebrachten Befürchtungen zerstreuen, dass das Gelehrte Journal vielleicht schon bald nach seiner Entstehung wegen geringer finanzieller Rentabilität eingestellt werden müsse. ${ }^{17}$

Von Gottsched wird erwartet, sofern er sich an der Societas incognitorum und den MA beteiligt, dass er als Berater fungiert, der auf wichtige Bücher auf dem (Wissenschafts-)Markt hinweisen soll, wobei Petrasch auch auf Hinweise bezüglich Neuerscheinungen aus dem französischen und englischen Raum zu hoffen scheint. Dem Eingeladenen stellt er - im Falle einer Zusage - ein Mitgliedsdiplom in Aussicht, verlangt aber noch ein Werkverzeichnis von Gottsched und fragt nach dessen Engagement im „Neuen Büchersaal der schönen Wissenschaften und freyen Künste“. ${ }^{18}$

Der Antwortbrief auf dieses Schreiben aus Olmütz ist leider nicht erhalten, muss jedoch positiv ausgefallen sein, da Gottsched in der Folge als Mitglied der Societas incognitorum angeführt wird und sich, insbesondere im dritten Band der MA, nicht nur

15 S. dazu Gebhardi, Ludwig Albrecht (1797): Geschichte aller Wendisch-Slavischen Staaten. 4. Band: Mähren und Schlesien. Halle: Gebauer, S. 185.

16 Köhler, Caroline/ Menzel, Franziska/ Otto, Rüdiger/ Schlott, Michael (Hrsg.) (2018): Johann Christoph Gottsched. Briefwechsel unter dem Einschluß des Briefwechsels von Luise Adelgunde Victorie Gottsched, Band 12: Oktober 1746-Dezember 1747. Berlin/ Boston: De Gruyter, S. 63.

17 S. Eybl, Franz (2015): Das Zeitalter des Buches. Buch-und Verlagswesen im Barock. In: Gastgeber, Christian/ Klecker, Elisabeth (Hrsg.): Barock. Graz: Akademische Druck-und Verlagsanstalt (Geschichte der Buchkultur, Band 6), S. 75-91.

18 Zur umfangreichen Tätigkeit Gottscheds als Herausgeber von Zeitschriften im Allgemeinen und zum „Büchersaal“ im Speziellen s. Ball, Gabriele (2000): Moralische Küsse. Gottsched als Zeitschriftenherausgeber und literarischer Vermittler. Göttingen: Wallstein Verlag, S. 123 f. 
als Verfasser eines umfangreichen Artikels zur Geschichte der österreichischen Literatur, sondern auch als Betreuer der Herausgabe des Gelehrten Journals vor Ort, also in Leipzig und Frankfurt, engagiert; nach immer weiter zunehmenden Problemen mit der Drucklegung in Olmütz, auf die hier nicht weiter eingegangen werden kann, mussten die MA ins „Ausland“ ausweichen, wo es bald (in Wechselwirkung mit der allmählichen Auflösung der Societas incognitorum) zur Einstellung des Periodikums kommt; das Ende der Societas incognitorum folgt bald darauf, im Jahr 1751.

\section{Zu Gottscheds Darstellung der Entwicklung der österreichischen Dichtkunst}

Im dritten Band der MA wurde ein Beitrag gekennzeichnet mit Gottscheds Namen publiziert, was eine Ausnahme darstellt, da die MA ansonsten durchgehend Artikel beinhaltet hatten, die anonym präsentiert wurden. Gottscheds erster ausführlicher „direkter Eingriff“ in die MA hatte wohl mehrere Gründe: Erstens befand sich das Gelehrte Journal (das nie als Literaturblatt intendiert gewesen war), wie bereits erwähnt, nunmehr mit der Verlegung des Druckortes in seiner Betreuung, und zweitens versuchte Gottsched Ende der 1740er Jahre, Anhänger und Unterstützer für seine Ideen, besonders für seinen Plan der Errichtung einer deutschen Akademie, in den österreichischen Länder zu gewinnen. ${ }^{19}$ Über die Societas incognitorum hatte er bereits einige zusätzliche Kontakte gewonnen, die bei der Umsetzung seines Vorhabens in Wien hätten hilfreich werden können; was lag da näher, sich auch - in einem österreichischen Journal - als Kenner der österreichischen Literaturgeschichte vorzustellen. Damit einher ging die Notwendigkeit, eine solche eigenständige Entwicklung und Kontinuität bis zur Gegenwart gewissermaßen zu erfinden und einen Ausblick für die Zukunft der Poesie deutscher Zunge in den österreichischen Ländern zu wagen. Den Hoffnungsträger hierfür sah er in einem seiner loyalsten Wiener „Gewährsmänner“, dem Verfasser der „Theresiade“ Franz Christoph Scheyb. ${ }^{20}$ Den Sekretär der niederösterreichischen Landesregierung positionierte er in seinem Artikel am Ende einer beinahe ununterbrochenen Reihe von Autoren, die seit dem Mittelalter in den österreichischen Ländern Literatur gewirkt hatten.

In der Aufzählung, die der Leipziger Professor für Poetik präsentiert, will er sich auf solche Dichter konzentrieren, „die sich in deutscher Sprache hervor gethan haben“21 - und beginnt im 13. Jahrhundert beim sagenhaften Meister Klingsor, den er als historische Persönlichkeit vorstellt, die später wegen ihrer erstaunlichen naturwissenschaft-

19 In Berlin war von Kurfürst Friedrich III. Bereits im Jahr 1700 eine Akademie gegründet worden, die ab 1744 den Titel einer „Königlichen Akademie der Wissenschaften“ trug. Zur Entwicklung der preußischen Akademie und ihrer Bedeutung im europäischen Wissenschaftsbetrieb s. Wiggermann, Uta (2010): Woellner und das Religionsedikt. Kirchenpolitik und kirchliche Wirklichkeit im Preußen des späten 18. Jahrhunderts. Tübingen; Mohr Siebeck Verlag (Beiträge zur historischen Theologie), S. 44.

20 Zu Scheybs Biographie vgl. Klemme, Heiner F./ Kuehn, Manfred (Hrsg.) (2010): The Bloomsbury Dictionary. Eighteenth-Century German Philosophers. New York: Bloomsbury Academic, S. 660.

21 MA, 3. Band, 2. Stück, S. 157. 
lichen Kenntnisse verleumdet worden sei. Ottokar von Hornecks „Österreichische Verschronik" verweist er auch in Bezug auf die Thematisierung des Zuges Ottokars II. nach Böhmen (dazu präsentiert er im Artikel auch einen Textausschnitt) und unterstreicht damit einmal mehr in seiner Darstellung die Bedeutung der böhmischen Länder im Rahmen der österreichischen Literaturentwicklung. ${ }^{22}$ Die Sprache Ottokar von Hornecks allerdings kritisiert Gottsched, indem er hervorhebt, dass Heinrich der Teichner eine „bessere Mundart als ein Steyrer“23 gebraucht habe.

Mit Peter Suchenwirth und einem unbekannten böhmischen Autor setzt er die Reihe fort und macht nach dem Tod von „Augustin von Hammersten“24 eine Lücke aus, die erst im 17. Jahrhundert langsam wieder geschlossen werden konnte; dabei hätten die deutschen Sprachgesellschaften, insbesondere die „Fruchtbringende Gesellschaft“ und der „Pegnesische Blumenorden“ eine bedeutende Rolle gespielt, wie der Autor des Artikels betont. ${ }^{25}$ Auf diese Weise bettet er seine Vision einer österreichischen Literaturentwicklung in die breite deutsche Literaturlandschaft ein und zeigt nicht nur diachrone Zusammenhänge, sondern auch die synchron wirksamen Dynamiken, die diese anscheinend von jeher beeinflusst hatten. Den letzten wichtigen Vorläufer Scheybs sieht er schließlich in Wolf Helmhard Freiherr von Hohberg, einem in Krems geborenen protestantischen Soldaten und Schriftsteller, der ebenfalls ein der habsburgischen Dynastie gewidmetes Werk, den „Habsburgischen Ottobert“, verfasst hatte. ${ }^{26}$

\section{Gottscheds Einfluss auf den Sprachgebrauch in den MA}

Eine bisher wenig beachtete Besonderheit der MA kann auch darin gesehen werden, dass sich die Societas incognitorum resp. die Redaktion des Gelehrten Journals, das sie hervorbrachte, im „barocken Sprachenstreit“27 gegen die oberdeutsche Schreibsprache stellte und sich für die „sächsische Norm“28 entschied, die Gottsched propagierte. Diese Entscheidung fiel damit einige Zeit bevor es in den habsburgischen Ländern

22 Über Horneck hatte der Benediktinermönch Hieronymus Pez, ebenfalls ein Mitglied der Societas incognitorum, bereits geforscht und publiziert; Gottsched schöpft mit ziemlicher Sicherheit aus dessen Ergebnissen. Diesen schließt sich in seiner Darstellung der österreichischen Wissenschaftslandschaft bis zum 18. Jahrhundert auch Khautz an, s. Khautz, Franz Constantin Florian (von) (1755): Geschichte der Oesterreichischen Gelehrten. Frankfurt und Leipzig: Bey Johann Friedrich Jahn, S. 19-26.

23 MA, 3. Band, 2. Stück, S. 158.

24 MA, 3. Band, 2. Stück, S. 182.

25 Zur Bedeutung der Sprachgesellschaften s. Otto, Karl (1972): Die Sprachgesellschaften des 17. Jahrhunderts. Stuttgart; Metzler Verlag, S. 17-18.

26 Zur Person Hohbergs bzw. seinem schriftstellerischen Werk s. auch Meyer, Torsten (2009): Cultivating the Landscape. The Perception and Description of Work in Sixteenth- and Eighteenth-Century German „Household Literature“ (Hausväterliteratur). In: Ehmer, Josef/ Lis, Catharina (Hrsg.): The Idea of Work in Europe from Antiquity to Modern Times. Farnham: Routledge, S. 215-245.

27 S. zur Vorgeschichte des Konfliktes und zum komplexen Verlauf der Diskussion Moser, Hugo (1968): Annalen der deutschen Sprache. Von den Anfängen bis zur Gegenwart. Stuttgart: Metzler Verlag, S. 43 f.

28 Ebd., S. 43. 
zu einer amtlichen Regelung bezüglich der zu verwendenden Kanzleisprache kommen sollte. ${ }^{29}$

Die Zusammensetzung der Societas incognitorum, in der auch einige Jesuiten und Benediktiner vertreten waren, die allgemein meist die oberdeutsche Variante bevorzugten, würde auf den ersten Blick, ebenso wie die geographische Lage (in der Provinz eines „süddeutschen“ Reichgebildes), vermuten lassen, dass sich die Gelehrten um Petrasch für die Variante von Johann Siegmund Popowitsch oder des Benediktinermönchs Augustin Dornblüths entscheiden würden. ${ }^{30}$ In der Einleitung zum ersten Stück des ersten Bandes verteidigt man zunächst vor allem die Entscheidung, warum man sich überhaupt für das Deutsche anstelle des Lateinischen als Wissenschaftssprache entscheidet, um dann klarzustellen, welche Variante des Deutschen man verwenden wolle:

„Wir haben das Teutsche/ als unsere Mutter-Sprach erwählet/ aus eben der Ursache/ als die Franzosen die ihrige/ die Italianer/ die Engelländer/ und andere jeglicher die seinige zu erkiesen pflegen. Es ist wahr/ daß die Lateinische Sprach unter denen Gelehrten die gemeineste/ doch dagegen gewis/ daß die Ungelehrte unserer Wercke noch mehers bedörffen/ und ihnen in selbiger zu lesen zu verdrüßlich fallet. Ferners hat uns dazu bewogen die Nothwenigkeit/ in welcher wir unsere Länder gesehen sich auf die Ausarbeitung ihrer eigenen Sprache zu legen. Derohalben wir die Grosse Schule der Teutschen Sprach an der Pleisse und deroselbten MundArt so viel uns Anfängern möglich/ als die vollkommenste Vorschrifften nehmen/ und alles/ was selbte hierinnen zu unserer Verbesserung beytragen wollen/mit freundschafftlichen Dank annehmen werden.“31

Wie im Brief an Gottsched, in dem er gebeten wurde, der Olmützer Gelehrtengesellschaft beizutreten, äußert sich Petrasch bescheiden, aber selbstbewusst und hebt hervor, wie sehr die Verfasser der MA auf Hilfe von Erfahreneren angewiesen seien, nicht zuletzt, was den Gebrauch des Deutschen in den Artikeln anbelangt. Die „deutsche Sprache an der Pleiße“ dient dabei von Anfang an, und wohl schon vor der Entscheidung, Gottsched in die Gelehrtengesellschaft mit einzubeziehen, als Referenzpunkt, wobei man aber anscheinend beabsichtigte, eine eigene Ausdrucksform im Rahmen des gewählten Modells zu finden.

Die MA wurden in ihrer Entscheidung innerhalb der böhmischen Länder insofern bestätigt, als dass ab Ende der 1750er Jahre der Einfluss der Leipziger Sprachtheoretiker und Dichter durch die Unterrichtsaktivität Karl Heinrich Ritter von Seibts an der Karls-Universität in Prag zunahm. Dieser hatte selbst in Leipzig studiert und hielt seine

29 Zur Diskussion um eine neue „Gemeinsprache der Gebildeten“, die frei von Provinzialismen zu sein hätte und auf die zeitgenössische Sprachentwicklung reagieren müsse vgl. Bödeker, Hans Erich/ Gierl, Martin (Hrsg.) (2007): Jenseits der Diskurse. Aufklärungspraxis und Institutionenwelt in europäisch komparativer Perspektive. Göttingen: Vandenhoeck und Ruprecht, S. 87.

30 Scheyb war einer der erbittersten Gegner Popowitschs in Wien, wobei auch die Jesuiten vor Ort - obgleich aus anderen Beweggründen -sich gegen dessen Sprachentwurf stellten, vgl. zu diesem Thema Havinga, Anna D (2018): Invisibilising Austrian German. On the effect of linguistic prescriptions and educational reforms on writing practices in 18th-century Austria. Berlin/ Boston: De Gruyter, S. 68-69.

31 MA, 1. Band, 1. Stück, S. 24. 
Vorlesungen in deutscher Sprache. Neben der Universität, zu deren Dekan er 1794 ernannt wurde, oblag ihm nach der Aufhebung des Jesuitenordens auch die Leitung der Prager Gymnasien, was seine Auffassung hinsichtlich des idealen Sprachgebrauchs bzw. des Zweck von Sprache überhaupt für die heranwachsende intellektuelle Elite in der böhmischen Metropole nutzbar werden ließ. ${ }^{32}$

\section{Gottsched in Wien}

Zu dem Zeitpunkt, als Gottsched seinen Beitrag zur österreichischen Literaturentwicklung veröffentlicht, ist er bereits mehr als ein Jahr Mitglied einer Gelehrtengesellschaft in den österreichischen Ländern und hat über diese Gesellschaft, der bekanntlich einige einflussreiche Hofbeamte angehörten, Zugang zu einem nützlichen Netzwerk an Personen, die ihm potentiell dabei helfen konnten, weitere Projekte in Wien anschieben zu können. Worum es ihm hauptsächlich ging, war die Errichtung einer österreichischen Akademie der Wissenschaften im Herzen des Habsburgerreichs, an der er selbst wohl einen zentralen Posten einzunehmen hoffte.

Seine Kontakte mit Friedrich dem Großen, einem möglichen alternativen Partner für seine Projekte, hatten - in der Zeit des Siebenjährigen Krieges gegen die habsburgische Herrscherin - angeblich zu keinen weiteren Erfolgen geführt; Friedrich ließ sowohl Gottsched als auch Gellert bei seinen Aufenthalten in Leipzig zu sich kommen und bevorzugte eindeutig den Fabeldichter, mit dem er anscheinend auch die Abneigung gegenüber dem Werk des von Gottsched gefeierten Königsberger Professor für Poesie, Johann Valentin Pietsch, teilte:

„Gottsched las ihm [Friedrich; Ergänzung der Autorin] seine Übersetzung von Racines Iphigenie vor; Gellert mußte eine seiner Fabeln hersagen. Jene machte dem König keinen großen Eindruck; mit dieser war er zufrieden:, Das ist schön', sagte er zu Gellert; ,recht schön‘: Er hat so was Coulantes in Seinen Versen. Das verstehe ich alles: da hat mir aber Gottsched eine Uebersetzung der Iphigenia vorgelsen, ich habe das Französische dabei gehabt und kein Wort verstanden: sie haben mir noch einen Poeten den Pietsch gebracht, den habe ich weggeworfen-, ,Ihro Majestät, erwiderte Gellert: ,den werfe ich auch weg.' [...]

Als Gellert fort war, bemerkte Friedrich: ,Das ist ein ganz anderer Mann als Gottsched.' Und den nächsten Tag bei Tafel nannte er ihn den vernünftigsten unter allen deutschen Gelehrten.“"33

32 Zur Situation der Erneuerung des Bildungssystems an den habsburgischen Universitäten in der Aufklärung s. Hlobíl, Tomáš (2011): Die Ästhetik in den Universitätsreformen des Wiener Hofes und an den Universitäten zu Wien, Prag und Freiburg im Breisgau (1763-1805). In: Diaconu, Mădalina/ Ševčík, Miloš (Hrsg.): Aesthetics Revisited. Tradition and Perspectives in Austria and in the Czech Republic. Berlin/ Münster/ Wien/ Zürich/ London: LIT Verlag, S. 100-106.

33 Scherer, Wilhelm (1902): Geschichte der Deutschen Litteratur. Berlin: Weidmannsche Buchhandlung, S. 395 . 
Gottsched versuchte im Herbst 1749 also sein Glück in Wien, wo das Kaiserpaar sich im Jahr zuvor von seinen Fähigkeiten als Dramatiker hatte überzeugen dürfen, als der „Sterbende Cato“ im Hofburgtheater aufgeführt worden war. Franz Christoph Scheyb vermittelte den Gottscheds nach deren Ankunft in Wien weitere Kontakte bzw. bauten diese ein eigenes Netzwerk auf. Gottsched wurde sogar eine Audienz bei der Kaiserin gewährt, die - in den Augen des preußischen Ehepaars - überraschend locker und unkonventionell über die Bühne ging ${ }^{34}$; die Inszenierung der Bescheidenheit der Herrscherfamilie, von der Martus spricht, funktionierte bei diesen Besuchern also wie beabsichtigt. ${ }^{35}$ Sein Ziel, die Kaiserin von der Notwendigkeit einer Akademie für Sprache und Literatur nach französischem Vorbild oder zumindest einer Deutschen Gesellschaft in Wien zu überzeugen, erreichte er nicht; wie in Olmütz verhinderten die Macht der Jesuiten und die Zensur, sowie die allgemeinen Bedingungen des Buch- und Zeitschriftenmarktes in den habsburgischen Ländern, weitere effektive Aktivitäten in diese Richtung. ${ }^{36}$ Scheyb scheint ihn auf die Macht der katholischen Geistlichkeit in Wien, wie in der mährischen Provinz, hingewiesen zu haben; anscheinend verlangte man von Gottsched auch eine Konversion zum Katholizismus. ${ }^{37}$ Ein Jahr nach Beendigung des „Österreichischen Erbfolgekrieges“, in welchem Friedrich Schlesien und die Grafschaft Glatz zugesprochen bekommen hatte, war die Zeit für große kulturelle Innovationen und Investitionen noch nicht reif - die tatsächliche Errichtung einer österreichischen Akademie der Wissenschaften sollte sich gut weitere 100 Jahre hinziehen, als Kaiser Ferdinand I. und sein Staatskanzler Metternich endlich eine solche ins Leben riefen.

Gellert, mit fünfzehn Jahren Altersunterschied fast eine Generation jünger als der Autor des 1730 erschienen „Versuchs einer critischen Dichtkunst vor die Deutschen“, taucht übrigens ebenfalls im dritten Band der MA auf, in welchem dem Leser vorbildhaft einige seiner Fabeln vorgestellt werden. ${ }^{38}$ Petrasch hielt sich persönlich bei seinen eigenen literarischen Versuchen ${ }^{39}$ an die Vorgaben Gottscheds, der wohl weitere Dichter aus seinem Umkreis vorschlug, die das Lesepublikum dieses Journals kennenlernen sollte. Die MA hatten sich bis dahin nur wenig der Literatur gewidmet (und wenn, dann mit Autoren wie Scheyb und Pamer, österreichische Autoren mit explizit patriotischen Werken ausgewählt), es wäre also nicht korrekt, sie als ein Literaturblatt zu bezeichnen; dennoch schien es der Redaktion auch darum zu gehen, den literarischen Horizont des

34 Die Kaiserin soll sich im Zuge der Begegnung bei Gottsched für ihr schlechtes Deutsch entschuldigt haben, wie Tapié beschreibt, s. Tapié, Victor L. (1989): Maria Theresia. Die Kaiserin und ihr Reich. Graz/ Wien/ Köln: Verlag Styria, S. 198.

35 Vgl. Martus, Steffen (2015): Aufklärung. Das deutsche 18. Jahrhundert. Ein Epochenbild. Berlin: Rowohlt Verlag, S. 82 f.

36 Zur Gründung einer permanenten Zensurkommission unter Maria Theresia s. Bachleitner, Norbert (2017): Die literarische Zensur in Österreich 1751 bis 1848. Wien/ Köln/ Weimar: Böhlau Verlag, S. 47.

37 Vgl. Becker-Cantarino, Barbara (2005): German Literature of the Eighteenth Century. The Enlightenment and Sensibility. Rochester/ New York: Camden House (Camden House history of German literature; Band 5), S. 248.

38 MA, 3. Band, 4. Stück, S. 235-286.

39 Zum literarischen Werk des Freiherrn von Petrasch s. Freude, Felix (1916): Die Schaubühne des Freiherrn von Petrasch. Brünn: Felix Freude. 
(österreichischen und mährischen bzw. böhmischen) Lesers zu erweitern. Es ist also anzunehmen, dass Gottsched die Literaturempfehlungen gegeben hatte, wie es von Anfang an geplant war, und wie es sich nun, als die MA außerhalb der habsburgischen Länder in seinem Einzugsbereich produziert wurden, auch praktisch besser realisieren ließ.

Außer Ausschnitten aus Gellerts Werk werden schließlich Oden und Lieder von Friedrich von Hagedorn präsentiert. ${ }^{40}$ Dabei wird - ganz im Sinne eines Gelehrten Journals - nicht verabsäumt, die Gattungen in ihrem historischen Entwicklungskontext zu verorten, um daraus auch die Legitimität der Texte, die eventuell als zu unterhaltsam oder zu seicht von vornherein hätten abgelehnt werden können, abzuleiten.

Zusammenfassend kann festgehalten werden, dass Gottsched vom Anfang der Societas incognitorum und der MA an eine wesentliche Rolle gespielt hatte; er unterstützte die Initiativen Petraschs, einem seiner treuen Anhänger, dadurch, dass er seinen Namen als Mitglied zur Verfügung stellte, um die Seriosität der Projekte zu unterstreichen. Er war sowohl inhaltlich durch seine Empfehlungen mitbestimmend, indem er beeinflusste, welche Werke besprochen werden sollten, als auch sprachlich, da sich die MA an seinem stilistischen Vorbild orientieren wollten (und wohl auch sollten). Die Mitgliedschaft in der Societas incognitorum, und das Engagement in den MA nutzte er außerdem, um seine Kontakte in den österreichischen Raum auszubauen und eventuell größere Projekte, wie etwa das einer Akademie der Wissenschaften in Wien, verwirklichen zu können.

\section{Bibliographie}

\section{Primärliteratur}

Monathliche Auszüge Alt, und neuer Gelehrten Sachen. Verlegts Franz Anton Hirnle, mit hoher Obrigkeitl. Erlaubnuss, 1747.

Ollmützer Monathliche Auszüge Alt- und neuer Gelehrten Sachen. III. Band. Franckfurt und Leipzig, bey Peter Conrad Monath, Buchhändler in Wien, 1748.

\section{Sekundärliteratur}

Bachleitner, Norbert (2017): Die literarische Zensur in Österreich 1751 bis 1848. Wien - Köln Weimar: Böhlau Verlag.Ball, Gabriele (2000): Moralische Küsse. Gottsched als Zeitschriftenherausgeber und literarischer Vermittler. Göttingen: Wallstein Verlag.

Becker-Cantarino, Barbara (2005): German Literature of the Eighteenth Century. The Enlightenment and Sensibility. Rochester - New York: Camden House (Camden House history of German literature; Band 5)

Bödeker, Hans Erich/ Gierl, Martin (Hrsg.) (2007): Jenseits der Diskurse. Aufklärungspraxis und Institutionenwelt in europäisch komparativer Perspektive. Göttingen: Vandenhoeck und Ruprecht.

40 MA, 3. Band, 4. Stück, S. 269-285. 
D'Elvert, Christian (1884): Zur Geschichte des Deutschthums in Oesterreich-Ungarn, mit besonderer Rücksicht auf die slavisch-ungarischen Länder. Brünn: C. Winiker.

D'Elvert, Christian (1866): Zur mährischen Adelsgeschichte. XI. Die Freiherrn von Petrasch. S. 33-36. In: Notizen-Blatt der historisch-statistischen Section der kaiserlich-königlich mährisch-schlesischen Gesellschaft zur Beförderung des Ackerbaus, der Natur- und Landeskunde. Beilage der Mittheilungen. Brünn: Verlag des Vereines für die Geschichte Mährens und Schlesiens.

Eybl, Franz (2015): Das Zeitalter des Buches. Buch-und Verlagswesen im Barock. In: Gastgeber, Christian - Klecker, Elisabeth (Hrsg.): Barock. Graz: Akademische Druck-und Verlagsanstalt (Geschichte der Buchkultur, Band 6), S. 75-91.

Freude, Felix (1916): Die Schaubühne des Freiherrn von Petrasch. Brünn: Felix Freude.

Gebhardi, Ludwig Albrecht (1797): Geschichte aller Wendisch-Slavischen Staaten. 4. Band: Mähren und Schlesien. Halle: Gebauer.

Habel, Thomas (2007): Gelehrte Journale und Zeitungen der Aufklärung. Zur Entstehung, Entwicklung und Erschließung deutschsprachiger Rezensionszeitschriften des 18. Jahrhunderts. Bremen: Edition lumière (Presse und Geschichte. Neue Beiträge. Hrsg. von Holger Böning, Michael Nagel und Johannes Weber; Band 17)

Havinga, Anna D (2018): Invisibilising Austrian German. On the effect of linguistic prescriptions and educational reforms on writing practices in 18th-century Austria. Berlin/ Boston: De Gruyter

Hlobíl, Tomáš (2011): Die Ästhetik in den Universitätsreformen des Wiener Hofes und an den Universitäten zu Wien, Prag und Freiburg im Breisgau (1763-1805). In: Diaconu, Mădalina - Ševčík, Miloš (Hrsg.): Aesthetics Revisited. Tradition and Perspectives in Austria and in the Czech Republic. Berlin - Münster - Wien - Zürich - London: LIT Verlag, S. 100-106.

Khautz, Franz Constantin Florian (von) (1755): Geschichte der Oesterreichischen Gelehrten. Frankfurt und Leipzig: Bey Johann Friedrich Jahn.

Klemme, Heiner F. - Kuehn, Manfred (Hrsg.) (2010): The Bloomsbury Dictionary. Eighteenth-Century German Philosophers. New York: Bloomsbury Academic.

Kollmann, Vitězlav (1998): Societas incognitorum a olomoučtí zednáři. In: Zprávy Vlastivědného muzea v Olomouci, Nr. 276, Olomouc, S. 1-8.

Králík, Oldřich (1947): Olomoucká Societas incognitorum. Olomouc: Lidové závody tiskařské a nakladatelské.

Köhler, Caroline - Menzel, Franziska - Otto, Rüdiger - Schlott, Michael (Hrsg.) (2018): Johann Christoph Gottsched. Briefwechsel unter dem Einschluß des Briefwechsels von Luise Adelgunde Victorie Gottsched, Band 12: Oktober 1746-Dezember 1747. Berlin/ Boston: De Gruyter.

Martus, Steffen (2015): Aufklärung. Das deutsche 18. Jahrhundert. Ein Epochenbild. Berlin: Rowohlt Verlag.

Meyer, Torsten (2009): Cultivating the Landscape. The Perception and Description of Work in Sixteenth- and Eighteenth-Century German „Household Literature“ (Hausväterliteratur). In: Ehmer, Josef - Lis, Catharina (Hrsg.): The Idea of Work in Europe from Antiquity to Modern Times. Farnham: Routledge, S. 215-245.

Moser, Hugo (1968): Annalen der deutschen Sprache. Von den Anfängen bis zur Gegenwart. Stuttgart: Metzler Verlag.

Otto, Karl (1972): Die Sprachgesellschaften des 17. Jahrhunderts. Stuttgart: Metzler Verlag.

Rieck, Werner (1973): Gottsched und die Societas incognitorum in Olmütz. In: Deutsche Akademie der Wissenschaften Berlin, Ost (Hrsg.): Forschungen und Fortschritte, Nr. 40. Berlin: Akademie Verlag, S. 83-85.

Sandys, John Edwin (2010): A History of Classical Scholarship. Charleston: Nabu Press. 
Scherer, Wilhelm (1902): Geschichte der Deutschen Litteratur. Berlin: Weidmannsche Buchhandlung.

Schram, Wilhelm (1894): Josef Freiherr von Petrasch und die „Gelehrte Gesellschaft der Unbekannten in Olmütz“. In: Notizenblatt des Vereines für die Geschichte Mährens und Schlesiens, Nr. 10, Brünn: Verlag des Vereines für die Geschichte Mährens und Schlesiens, S. 89-91.

Tapié, Victor L. (1989): Maria Theresia. Die Kaiserin und ihr Reich. Graz/ Wien/ Köln: Verlag Styria.

Wiggermann, Uta (2010): Woellner und das Religionsedikt. Kirchenpolitik und kirchliche Wirklichkeit im Preußen des späten 18. Jahrhunderts. Tübingen: Mohr Siebeck Verlag (Beiträge zur historischen Theologie)

Wögerbauer, Michael - Pokorný, Jiří (2015): Barocke Buchkultur in den böhmischen Ländern. In: Gastgeber, Christian - Klecker, Elisabeth (Hrsg.) (2015): Barock. Graz: Akademische Druck-und Verlagsanstalt, S. 383-426.

Wondrák, Eduard (1972): Die olmützer „Societas incognitorum“. Zum 225. Jubiläum ihrer Gründung und zum 200. Todestag ihres Gründers. In: Ischreyt, Heinz u. A. (Hrsg.): Die Aufklärung in Ost- und Südosteuropa. Aufsätze, Vorträge, Dokumentationen. Köln/ Wien: Böhlau Verlag, S. 215-228.

Mag. Dr. Sabine Voda Eschgfäller / sabine.voda@upol.cz

Univerzita Palackého v Olomouci, Filozofická fakulta, Katedra germanistiky

Křižkovského 10, 77180 Olomouc, Česká republika 
